\title{
A review of sugar palm (Arenga pinnata): application, fibre characterisation and composites
}

\begin{abstract}
Purpose-The purpose of this paper is to present the review of natural fibre composites as well as a specific type of fibre, i.e., sugar palm fibre and its composites. Design/methodology/approach-The approach of this review paper is to present previous work on natural fibres and their composites. Then a review of several important aspects such as history, origin, botanic description, distribution, application and characterisation of sugar palm tree, and its fibre is presented. Finally a review of properties and characterisation of sugar palm composites is presented. Findings-Findings of this review include the potential application of natural fibres and their composites for engineering application, the use of sugar palm and its fibres, as well as the suitability of sugar palm composites in engineering application after conducting review of their performance and characterisation. Originality/value-The value of this review is to highlight the potential of natural fibres, natural fibre composites, sugar palm, sugar palm fibres and sugar palm composites as materials for engineering applications.
\end{abstract}

Keyword: Arenga pinnata; Bio-composites; Mechanical performance; Natural fibres; Sugar palm fibre; Sugar palm tree 